\title{
Similar Construction Method of Solution for Solving the Mathematical Model of Fractal Reservoir with Spherical Flow
}

\author{
Cui-Cui Sheng, ${ }^{1}$ Jin-Zhou Zhao, ${ }^{1}$ Yong-Ming Li, ${ }^{1}$ Shun-Chu Li, ${ }^{2}$ and Hu Jia ${ }^{1}$ \\ ${ }^{1}$ State Key Laboratory of Oil and Gas Reservoir Geology and Exploitation, Southwest Petroleum University, Chengdu 610500, China \\ ${ }^{2}$ Institute of Applied Mathematics, Xihua University, Chengdu 610039, China \\ Correspondence should be addressed to Shun-Chu Li; lishunchu@163.com
}

Received 28 February 2013; Accepted 8 May 2013

Academic Editor: Kazutake Komori

Copyright (c) 2013 Cui-Cui Sheng et al. This is an open access article distributed under the Creative Commons Attribution License, which permits unrestricted use, distribution, and reproduction in any medium, provided the original work is properly cited.

\begin{abstract}
On the basis of similar structure of solution for a second-order linear differential equation's boundary value problem, and our analysis of the relationship between this similar structure and its kernel function, the differential equation, and the boundary conditions, we propose a new simple solution-similar constructive method of solution (SCMS)—and sum up its detailed steps. We set up a porous media model under three kinds of outer boundary conditions (infinite, constant pressure, and closed), in which the influences of fractal dimension, spherical flow, skin effect, and storage are taken into consideration. And then we use SCMS to solve it. The SCMS is a straightforward method for differential equation's boundary value problems with complex boundary conditions, especially for solving the reservoir models in petroleum engineering.
\end{abstract}

\section{Introduction}

In the early 21 st century, Jia and Li [1] put forward an important hypothesis that the solutions of differential equations under different boundary conditions have similaritiessimilar structure of solutions, just as a real number can be expressed in continued fraction and geometric graphic have certain similarity. After that, many studies have been carried out on the similar structure of solutions for some boundary value problems of second-order linear homogeneous differential equations [2-8].

In the past two decades, many researchers have studied the fractal nature of reservoir rocks and other porous media. Fractal theory can be applied to effectively describe the complexities of a real reservoir, especially of the naturally fractured reservoir or heterogeneous media. Chang and Yortsos [9] were the first to apply the fractal theory to reservoir model, and then set up the mathematical model of fractal reservoir. Beier [10] afterwards extended the fractal model to the study of hydraulically fractured well, and discovered a power-law behavior during the linear and radial flow period. Camacho-Velázquez et al. [11] successfully applied the fractal model to explore the naturally fractured reservoir and obtained a series of decline curves. Using the same model, Cossio [12] proposed a semianalytic solution for finite-conductive flow in vertical fractures, and from the expression of the solution, people can easily perceive the relationship between the fractal parameters $\left\{d_{f}, \theta\right\}$ and the pressure waves or streamlines. Li et al. [13-16] built mathematical models for fluid flow in porous media, such as homogeneous reservoir mathematical model, double porosity reservoir mathematical model, multilayer reservoir mathematical model, and composite reservoir mathematical model. They found that under three kinds of outer boundary conditions (infinite, constant pressure, and closed), their pressure can also be expressed in similar structures in the Laplace space.

When a reservoir is mined with a small opening in the upper reservoir, or the thickness of reservoir stratum is larger than the radius of well bore, there still exists the vertical flow in the flow region, so it cannot be considered as cylindrical flow. Ershaghi et al. [17] analyzed pressure transient data in reservoirs with natural fractures and spherical flow. He found out that the cylindrical flow could not be applied to these reservoirs, or there would be large errors. Proett and Chin [18] provided a new exact spherical flow solution with storage and skin taken into consideration in early-time interpretation 
with applications to wire line formation and early-evaluation drill stem testing. In recent years, we [19-23] have built some mathematical models for reservoir with spherical flow, and through a complicated process of solution, we obtained their expressions of pressure in the Laplace space.

Cheng et al. [24, 25] put forward the idea of SCMS, but they did not specify its detailed procedures. Based on their research, the SCMS is extended in our paper in detail. In this paper, the first two sections focus on solving the boundary value problem of a second-order linear differential equation. Its solution can be expressed in a continued fraction. On the basis of the relationship among similar structure of solution, its kernel function, differential equation, and boundary conditions, we sum up the detailed construction steps of SCMS. In this paper, we also built a new fractal mathematical model for the reservoirs with spherical flow. To apply SCMS to solve this reservoir model, in Section 3 we first study a boundary value problem of the modified Bessel equation, which frequently occurred in reservoir engineering. So we could conveniently construct formation pressure and wellbore pressure. Through the expressions of formation pressure and wellbore pressure and their kernel functions, we could analyze the influences of the variable flow rate, the wellbore storage, the skin effect, the fractal dimension, and the fractal index on them. In a word, SCMS is a straightforward method for boundary value problems of differential equations with complex boundary conditions, especially for solving the reservoir models in petroleum engineering.

\section{Similar Structure of Solution for the Boundary Value Problem of a Second-Order Linear Differential Equation}

The boundary value problem of a second-order linear differential equation:

$$
\begin{gathered}
y^{\prime \prime}+p(x) y^{\prime}+q(x) y=0, \\
{\left[a y+(1+a b) y^{\prime}\right]_{x=\alpha}=c,} \\
{\left[e y+f y^{\prime}\right]_{x=\beta}=0,}
\end{gathered}
$$

where $p(x), p^{\prime}(x)$, and $q(x)$ are continuous function within $[\alpha, \beta] . a, b, c, e, f$ are real numbers. When $p(x)>0, q(x) \geq 0$ and $q(x)$ is not constantly zero, and $a(1+a b) \leq 0$, ef $\geq 0$, $a^{2}+(1+a b)^{2} \neq 0, e^{2}+f^{2} \neq 0,0<\alpha<\beta$, this boundary value problem has a unique solution [26]; this solution can be expressed in the following continued fraction:

$$
y(x)=c \cdot \frac{1}{a+1 /(b+\phi(\alpha))} \cdot \frac{1}{b+\phi(\alpha)} \cdot \phi(x),
$$

where $\phi(x)$ is a similar kernel function and defined as

$$
\phi(x)=\frac{e \varphi_{0,0}(x, \beta)+f \varphi_{0,1}(x, \beta)}{e \varphi_{1,0}(\alpha, \beta)+f \varphi_{1,1}(\alpha, \beta)} .
$$

We assume that $y_{1}(x)$ and $y_{2}(x)$ are the linearly independent solutions of the differential equation (1); then we define a binary function and calculate its partial derivative for $x_{1}, x_{2}$ respectively, listed as follows:

$$
\begin{aligned}
& \varphi_{0,0}\left(x_{1}, x_{2}\right)=y_{1}\left(x_{1}\right) y_{2}\left(x_{2}\right)-y_{1}\left(x_{2}\right) y_{2}\left(x_{1}\right), \\
& \varphi_{0,0}(x, \beta)=y_{1}(x) y_{2}(\beta)-y_{1}(\beta) y_{2}(x), \\
& \varphi_{0,1}(\alpha, \beta)=\left.\frac{\partial}{\partial x_{2}} \varphi_{0,0}\left(x_{1}, x_{2}\right)\right|_{(\alpha, \beta)} \\
&=y_{1}(\alpha) y_{2}^{\prime}(\beta)-y_{1}^{\prime}(\beta) y_{2}(\alpha), \\
& \varphi_{1,0}(\alpha, \beta)=\left.\frac{\partial}{\partial x_{1}} \varphi_{0,0}\left(x_{1}, x_{2}\right)\right|_{(\alpha, \beta)} \\
&=y_{1}^{\prime}(\alpha) y_{2}(\beta)-y_{1}(\beta) y_{2}^{\prime}(\alpha) \\
& \varphi_{1,1}(\alpha, \beta)=\left.\frac{\partial}{\partial x_{1}} \varphi_{0,1}\left(x_{1}, x_{2}\right)\right|_{(\alpha, \beta)} \\
&=\left.\frac{\partial}{\partial x_{2}} \varphi_{1,0}\left(x_{1}, x_{2}\right)\right|_{(\alpha, \beta)} \\
&=y_{1}^{\prime}(\alpha) y_{2}^{\prime}(\beta)-y_{1}^{\prime}(\beta) y_{2}^{\prime}(\alpha) .
\end{aligned}
$$

\section{Similar Construction Method of Solution (SCMS)}

On the basis of the relationship among the similar structure of solution (4), its similar kernel function (5), and boundary conditions (2)-(3), the SCMS steps are presented as below.

Step 1 . Solve the linearly independent solutions $y_{1}(x)$ and $y_{2}(x)$ of the differential equation (1).

Step 2. Construct a binary function $\varphi_{0,0}\left(x_{1}, x_{2}\right)$ with $y_{1}(x)$ and $y_{2}(x)$ (e.g., (6)) and calculate its partial derivative for $x_{1}$, $x_{2}$, respectively (e.g., (7)-(10)).

Step 3. Construct a similar kernel function $\phi(x)$, (e.g., (5)) with the coefficients of the right boundary condition (3) and the binary function $\varphi_{0,0}\left(x_{1}, x_{2}\right)$ and its partial derivative for $x_{1}, x_{2}$.

Step 4. Construct similar structure of solution (4) with the similar kernel function $\phi(x)$ and the coefficients of the left boundary condition (2).

\section{SCMS for Solving the Boundary Value Problem of the Modified Bessel Equation}

To apply SCMS to solve the reservoir model, we first introduce the boundary value problem of the modified Bessel 
equation, which frequently occurred in reservoir engineering:

$$
\begin{gathered}
x^{2} y^{\prime \prime}+A x y^{\prime}+\left(E-F x^{d^{\prime}}\right) y=0, \\
{\left[a^{\prime} y+\left(1+a^{\prime} b^{\prime}\right) y^{\prime}\right]_{x=\alpha^{\prime}}=c^{\prime},} \\
{\left[e^{\prime} y+f^{\prime} y^{\prime}\right]_{x=\beta^{\prime}}=0,}
\end{gathered}
$$

where $a^{\prime}, b^{\prime}, c^{\prime}, d^{\prime}, e^{\prime}, f^{\prime}, \alpha^{\prime}, \beta^{\prime}, A, E$, and $F$ are real numbers. And they meet the conditions

$$
\begin{array}{cc}
(1-A)^{2} \geq 4 E, \quad F>0, & a^{\prime}\left(1+a^{\prime} b^{\prime}\right) \leq 0, \\
e^{\prime} f^{\prime} \geq 0, \quad d^{\prime} \neq 0, & a^{\prime 2}+\left(1+a^{\prime} b^{\prime}\right)^{2} \neq 0, \\
\left(e^{\prime}\right)^{2}+\left(f^{\prime}\right)^{2} \neq 0, & 0<\alpha^{\prime}<\beta^{\prime} .
\end{array}
$$

According to SCMS in Section 3, we can construct the similar structure and its similar kernel functions of the solutions of the boundary value problem (11)-(13).

Step 1. The linearly independent solutions of the modified Bessel equation (11) are as follows (with details in Appendix A):

$$
x^{(1-A) / 2} I_{\nu}\left(\frac{2 \sqrt{F}}{d^{\prime}} x^{d^{\prime} / 2}\right), \quad x^{(1-A) / 2} K_{\nu}\left(\frac{2 \sqrt{F}}{d^{\prime}} x^{d^{\prime} / 2}\right),
$$

where $I_{i}(\cdot)$ denotes the first type of the modified Bessel function with order $i, K_{i}(\cdot)$ denotes the second type of the modified Bessel function with order $i$, and $v=\sqrt{(1-A)^{2}-4 E} / d^{\prime}$.

Step 2. Construct a binary function:

$$
\begin{aligned}
M_{0,0}(x, \tau)= & x^{(1-A) / 2} K_{\nu}\left(\frac{2 \sqrt{F}}{d^{\prime}} x^{d^{\prime} / 2}\right) \\
& \times \tau^{(1-A) / 2} I_{\nu}\left(\frac{2 \sqrt{F}}{d^{\prime}} \tau^{d^{\prime} / 2}\right) \\
& -\tau^{(1-A) / 2} K_{\nu}\left(\frac{2 \sqrt{F}}{d^{\prime}} \tau^{d^{\prime} / 2}\right) \\
& \times x^{(1-A) / 2} I_{\nu}\left(\frac{2 \sqrt{F}}{d^{\prime}} x^{d^{\prime} / 2}\right) \\
= & (x \tau)^{(1-A) / 2} \psi_{\nu, \nu}\left(x^{d^{\prime} / 2}, \tau^{d^{\prime} / 2}, \frac{2 \sqrt{F}}{d^{\prime}}\right),
\end{aligned}
$$

where

$$
\begin{aligned}
\psi_{m, n}\left(x^{\varepsilon}, y^{\varepsilon}, \xi\right)= & K_{m}\left(\xi x^{\varepsilon}\right) I_{n}\left(\xi y^{\varepsilon}\right) \\
& +(-1)^{m-n+1} I_{m}\left(\xi x^{\varepsilon}\right) K_{n}\left(\xi y^{\varepsilon}\right),
\end{aligned}
$$

where $m$ and $n$ are real numbers.
Then, calculate the binary function's partial derivatives for $x, \tau$ :

$$
\begin{aligned}
M_{0,1}(x, \tau)= & \frac{\partial}{\partial \tau} M_{0,0}(x, \tau) \\
= & x^{(1-A) / 2} \tau^{-(1+A) / 2} \\
& \times\left[\frac{1-A+\sqrt{(1-A)^{2}-4 E}}{2}\right. \\
& \quad \times \psi_{\nu, \nu}\left(x^{d^{\prime} / 2}, \tau^{d^{\prime} / 2}, \frac{2 \sqrt{F}}{d^{\prime}}\right) \\
& \left.\quad+\sqrt{F} \tau^{d^{\prime} / 2} \psi_{\nu, \nu+1}\left(x^{d^{\prime} / 2}, \tau^{d^{\prime} / 2}, \frac{2 \sqrt{F}}{d^{\prime}}\right)\right],
\end{aligned}
$$$$
M_{1,0}(x, \tau)=\frac{\partial}{\partial x} M_{0,0}(x, \tau)
$$$$
=x^{-(1+A) / 2} \tau^{(1-A) / 2}
$$$$
\times\left[\frac{1-A+\sqrt{(1-A)^{2}-4 E}}{2}\right.
$$

$$
\begin{aligned}
& \times \psi_{\nu, \nu}\left(x^{d^{\prime} / 2}, \tau^{d^{\prime} / 2}, \frac{2 \sqrt{F}}{d^{\prime}}\right) \\
& \left.-\sqrt{F} x^{d^{\prime} / 2} \psi_{\nu+1, \nu}\left(x^{d^{\prime} / 2}, \tau^{d^{\prime} / 2}, \frac{2 \sqrt{F}}{d^{\prime}}\right)\right],
\end{aligned}
$$

$$
\begin{aligned}
M_{1,1}(x, \tau)= & \frac{\partial}{\partial x} M_{0,1}(x, \tau)=\frac{\partial}{\partial \tau} M_{1,0}(x, \tau) \\
= & (x \tau)^{-(1+A) / 2} \\
& \times\left\{\frac{\left[1-A+\sqrt{(1-A)^{2}-4 E}\right]^{2}}{2}\right. \\
& \times \psi_{\nu, \nu}\left(x^{d^{\prime} / 2}, \tau^{d^{\prime} / 2}, \frac{2 \sqrt{F}}{d^{\prime}}\right) \\
& +\frac{\sqrt{F}\left[1-A+\sqrt{(1-A)^{2}-4 E}\right]}{2}
\end{aligned}
$$

$$
\times \tau^{d^{\prime} / 2} \psi_{\nu, v+1}\left(x^{d^{\prime} / 2}, \tau^{d^{\prime} / 2}, \frac{2 \sqrt{F}}{d^{\prime}}\right)
$$

$$
-\frac{\sqrt{F}\left[1-A+\sqrt{(1-A)^{2}-4 E}\right]}{2}
$$




$$
\begin{aligned}
& \times x^{d^{\prime} / 2} \psi_{\nu+1, \nu}\left(x^{d^{\prime} / 2}, \tau^{d^{\prime} / 2}, \frac{2 \sqrt{F}}{d^{\prime}}\right) \\
& \left.-F(x \tau)^{d^{\prime} / 2} \psi_{\nu+1, \nu+1}\left(x^{d^{\prime} / 2}, \tau^{d^{\prime} / 2}, \frac{2 \sqrt{F}}{d^{\prime}}\right)\right\} .
\end{aligned}
$$

Step 3. Construct similar kernel functions with the coefficients of the right boundary condition (13), the binary function (16), and its partial derivatives (18):

$$
\phi(x)=\frac{e^{\prime} M_{0,0}\left(x, \beta^{\prime}\right)+f^{\prime} M_{0,1}\left(x, \beta^{\prime}\right)}{e^{\prime} M_{1,0}\left(\alpha^{\prime}, \beta^{\prime}\right)+f^{\prime} M_{1,1}\left(\alpha^{\prime}, \beta^{\prime}\right)} .
$$

Step 4. Construct the similar structure of solution with the similar kernel function (19) and coefficients of the left boundary condition (12):

$$
y(x)=c^{\prime} \cdot \frac{1}{a^{\prime}+1 /\left(b^{\prime}+\phi\left(\alpha^{\prime}\right)\right)} \cdot \frac{1}{b^{\prime}+\phi\left(\alpha^{\prime}\right)} \cdot \phi(x) .
$$

Consequently, (20) is the solution of the boundary value problem of the modified Bessel equation (11)-(13), which is similar to the real numbers and can be expressed as continued fraction.

\section{SCMS for Solving the Mathematical Model of Fractal Reservoir with Spherical Flow}

In Appendix B, the dimensionless mathematical model of fractal reservoir with spherical flow is presented. It takes dimensionless pressure as variable, and accords with Darcy flow. The details are as follows.

Fundamental differential equation:

$$
\frac{\partial^{2} p_{D}}{\partial r_{D}^{2}}+\frac{\zeta}{r_{D}} \frac{\partial p_{D}}{\partial r_{D}}=\frac{r_{D}^{\theta}}{e^{2 S}} \frac{\partial p_{D}}{\partial t_{D}}, \quad r_{D}>1, t_{D}>0,
$$

where:

$$
\zeta=d_{f}-d-\theta+2
$$

Initial condition:

$$
p_{D}\left(r_{D}, 0\right)=0 \text {. }
$$

Inner boundary condition:

$$
\frac{d p_{w D}}{d r_{D}}=-q_{D}\left(t_{D}\right)+C_{D} \frac{d p_{w D}}{d t_{D}} .
$$

Three kinds of outer boundary conditions:

$$
p_{D}\left(\infty, t_{D}\right)=0, \quad p_{D}\left(R_{D}, t_{D}\right)=0,\left.\quad \frac{\partial p_{D}}{\partial r_{D}}\right|_{r_{D}=R_{D}}=0 .
$$

They denote that the outer boundaries of circular reservoir are infinite pressure, constant pressure, and closed, respectively.

All the notations are explained in the nomenclature section.

Taking the Laplace transform of $p_{D}\left(r_{D}, t_{D}\right)$ with respect to $t_{D}$ and using the initial condition (23), we get the boundary value problem of the ordinary differential equation with parameter $z$ (where $z$ is Laplace space variable); that is,

$$
\begin{gathered}
\frac{d^{2} \bar{p}_{D}}{d r_{D}^{2}}+\frac{\zeta}{r_{D}} \frac{d \bar{p}_{D}}{d r_{D}}=\frac{r_{D}^{\theta}}{e^{2 S}} z \bar{p}_{D}, \\
{\left.\left[-C_{D} z \bar{p}_{w D}\left(r_{D}, z\right)+\frac{d}{d r_{D}} \bar{p}_{D}\left(r_{D}, z\right)\right]\right|_{r_{D}=1}=-\bar{q}_{D}(z),} \\
\bar{p}_{D}(\infty, z)=0 \text { or } \bar{p}_{D}\left(R_{D}, z\right)=0 \text { or }\left.\frac{d \bar{p}_{D}}{d r_{D}}\right|_{r_{D}=R_{D}}=0 .
\end{gathered}
$$

With reference to SCMS in Section 3 and its comparison with the boundary value problem of the modified Bessel equation, we can infer

$$
\begin{gathered}
y=\bar{p}_{D}, \quad x=r_{D}, \quad A=\zeta, \quad E=0, \quad F=\frac{z}{e^{2 S}}, \\
a^{\prime}=-C_{D} z, \quad b^{\prime}=0, \quad c^{\prime}=-\bar{q}_{D}(z), \\
\alpha^{\prime}=1, \quad v=\frac{\zeta-1}{2+\theta}
\end{gathered}
$$

and setting $e^{\prime}=1, f^{\prime}=0, \beta^{\prime} \rightarrow \infty$ or $e^{\prime}=1, f^{\prime}=0, \beta^{\prime}=$ $R_{D}$ or $e^{\prime}=0, f^{\prime}=1, \beta^{\prime}=R_{D}$ represent the three kinds of outer boundary conditions, respectively. So we can construct the similar structure of solution of the dimensionless mathematical model of fractal reservoir with spherical flow (26):

$$
\begin{aligned}
& \bar{p}\left(r_{D}, z\right) \\
& \quad=-\bar{q}_{D}(z) \cdot \frac{1}{-C_{D} z+1 / \phi(1, z)} \cdot \frac{1}{\phi(1, z)} \cdot \phi\left(r_{D}, z\right),
\end{aligned}
$$

where $\phi\left(r_{D}, z\right)$ is also a similar kernel function. Its expression is the following.

$$
\text { For } \bar{p}_{D}(\infty, z)=0 \text {, }
$$

$$
\begin{aligned}
& \phi\left(r_{D}, z\right) \\
& =-\frac{K_{\nu}\left((2 /(2+\theta)) \sqrt{z / e^{2 S}} r_{D}^{(2+\theta) / 2}\right)}{r_{D}^{\theta / 2} \sqrt{z / e^{2 S}}\left[K_{\nu+1}\left((2 /(2+\theta)) \sqrt{z / e^{2 S}} r_{D}^{(2+\theta) / 2}\right)\right]} .
\end{aligned}
$$

In (29), we use the properties of $\lim _{x \rightarrow \infty} I_{i}(x)=\infty$ and $\lim _{x \rightarrow \infty} K_{i}(x)=0$ to obtain the result above. 


$$
\begin{aligned}
& \text { For } \bar{p}_{D}\left(R_{D}, z\right)=0, \\
& \phi\left(r_{D}, z\right) \\
& \quad=-\frac{\psi_{\nu, v}\left(r_{D}^{(2+\theta) / 2}, R_{D}^{(2+\theta) / 2},(2 /(2+\theta)) \sqrt{z / e^{2 S}}\right)}{\sqrt{z r_{D}^{\theta} / e^{2 S}} \psi_{\nu+1, \nu}\left(r_{D}^{(2+\theta) / 2}, R_{D}^{(2+\theta) / 2},(2 /(2+\theta)) \sqrt{z / e^{2 S}}\right)} .
\end{aligned}
$$

For $d \bar{p}_{D} /\left.d r_{D}\right|_{r_{D}=R_{D}}=0$,

$$
\begin{aligned}
& \phi\left(r_{D}, z\right) \\
& \quad=-\frac{\psi_{\gamma, v+1}\left(r_{D}^{(2+\theta) / 2}, R_{D}^{(2+\theta) / 2},(2 /(2+\theta)) \sqrt{z / e^{2 S}}\right)}{\sqrt{z r_{D}^{\theta} / e^{2 S}} \psi_{\nu+1, \nu+1}\left(r_{D}^{(2+\theta) / 2}, R_{D}^{(2+\theta) / 2},(2 /(2+\theta)) \sqrt{z / e^{2 S}}\right)} .
\end{aligned}
$$

Thus, (28) is the similar structure of formation pressure of the fractal reservoir with spherical flow in the Laplace space.

Then, setting $r_{D}=1$, we can obtain the similar structure of wellbore pressure of the fractal reservoir with spherical flow in the Laplace space:

$$
\bar{p}_{w}(z)=-\bar{q}_{D}(z) \cdot \frac{1}{-C_{D} z+1 / \phi(1, z)}
$$

Substituting (29), (30), and (31) into (28) and (32), respectively, we can get the expressions of formation pressures and the wellbore pressures of thefractal reservoir with spherical flow under three kinds of boundary conditions in the Laplace space. Then, they can be inverted numerically from the Laplace space by using the Stehfest algorithm [27].

Note that the solution described above is a general spherical flow model. Its special case are constant production rate $\left(q(t)=q_{0}\right)$, no skin effect $(S=0)$, no wellbore storage $(C=0)$, and homogeneous reservoir $\left(d_{f}=0, \theta=0\right)$.

\section{Conclusions}

Based on the reasoning mentioned above, the following conclusions can be drawn.

(1) When dealing with the boundary value problem of a general second-order linear differential equation, we find that the kernel function is related to right (outer) boundary condition and similar structure of solution is related to left (inner) boundary condition. So the SCMS is proposed to solve such complicated problems in reality.

(2) Many reservoir models can be adapted to the boundary value problem of the modified Bessel equation. So SCMS is a convenient, effective, and creative method to solve the problem of fluid flow in porous media since it can avoid complex procedures. SCMS optimizes the programming process of well test analysis software and also simplifies the arithmetic process.

(3) The mathematical model of fractal reservoir with spherical flow is a new model. It is particularly useful when the thick reservoir is mined in an imperfect model. On the basis of the continued fractions (see (28) and (32)), we can directly analyze the wellbore storage's influence on the formation pressure and wellbore pressure. Based on (29)-(31), it is convenient to see how the fractal dimension, fractal exponent, skin effect, and the three outer boundary conditions influence the kernel functions. Then, substituting (29), (30), and (31) into (28) and (32), respectively, we can see the influence of these parameters on the formation pressure and wellbore pressure.

(4) After the pressure data is processed with the Laplace transformation, well test analysis can be conducted directly in the Laplace space, which can reflect the advantage of the similar structure of solutions. Finally, we can use the Stehfest algorithm to obtain the solution in real space, which meets the demand of well test analysis.

\section{Appendices}

\section{A. Derivation of Two Linearly Independent Solutions of the Modified Bessel Equation}

In this section, details are shown to solve the generalized solution of the modified Bessel equation (11).

Making the substitution

$$
h=x^{-(1-D) / 2} y
$$

into (11), we obtain

$$
\frac{d^{2} h}{d s^{2}}+\frac{1}{s} \frac{d h}{d s}-\left(1+\frac{v_{1}^{2}}{s^{2}}\right) h=0
$$

where

$$
s=\frac{2 \sqrt{F}}{d} x^{d / 2} .
$$

According to [28], the general solutions of (A.2) can be obtained to be

$$
h=A_{1} K_{\nu}\left(\frac{2 \sqrt{F}}{d} x^{d / 2}\right)+A_{2} I_{\nu}\left(\frac{2 \sqrt{F}}{d} x^{d / 2}\right),
$$

where

$$
v=\frac{\sqrt{(1-D)^{2}-4 E}}{d} .
$$

By substituting (A.1) into (A.4), the general solution of (11) is

$$
y(x)=x^{(1-D) / 2}\left[A_{1} K_{v}\left(\frac{2 \sqrt{F}}{d} x^{d / 2}\right)+A_{2} I_{\nu}\left(\frac{2 \sqrt{F}}{d} x^{d / 2}\right)\right] .
$$

We know that

$$
x^{(1-D) / 2} I_{\nu}\left(\frac{2 \sqrt{F}}{d} x^{d / 2}\right), \quad x^{(1-D) / 2} K_{\nu}\left(\frac{2 \sqrt{F}}{d} x^{d / 2}\right)
$$

are two linearly independent solutions for the modified Bessel equation (11). 


\section{B. Mathematical Model of Fractal Reservoir with Spherical Flow}

Here we demonstrate the derivation of the mathematical model for fractal reservoir with spherical flow. The main assumptions of the model include

(i) flow obeys Darcy's law and isothermal curve;

(ii) spherical flow which has one production or injection well;

(iii) formation pressure is initial reservoir pressure $p_{0}$ before producing. But the well produces or injects at a variable rate $q(t)$.

We consider skin effects by $r_{\text {we }}=r_{w} e^{-S}$, where $r_{w}$ is wellbore radius, $r_{\text {we }}$ is effective wellbore radius, and $S$ is skin factor $(S>0$, damaged; $S<0$, improved). Here, a simplified formula introduced by Chang and Yortsos [9], later refined by Acuña et al. [29], is used to describe the reservoir porosity and permeability as functions of distance:

$$
\begin{aligned}
& \phi(r)=\phi_{\mathrm{we}}\left(\frac{r}{r_{\mathrm{we}}}\right)^{d_{f}-d}, \\
& k(r)=k_{\mathrm{we}}\left(\frac{r}{r_{\mathrm{we}}}\right)^{d_{f}-d-\theta},
\end{aligned}
$$

where $d, d_{f}, \theta$, and $r$ are Euclid dimension, fractal dimension, conductivity index, and radial distance in spherical coordinate, respectively.

Darcy's law says

$$
v_{r}=-\frac{k}{\mu} \frac{\partial p}{\partial r},
$$

where $v_{r}$ is spherical flow velocity and $\mu$ is viscosity of fluid.

Due to the conservation law, the continuity equation is given by

$$
\frac{1}{r^{2}} \frac{\partial}{\partial r}\left(r^{2} \rho v_{r}\right)+\frac{\partial\left(\phi_{f} \rho\right)}{\partial t}=0 .
$$

Substituting (B.1)-(B.2) into (B.3), we obtain the fundamental differential equation accounting for the fractal reservoir with spherical flow:

$$
\frac{\partial^{2} p}{\partial r^{2}}+\frac{\zeta}{r} \frac{\partial p}{\partial r}=\left(\frac{r}{r_{\mathrm{we}}}\right)^{\theta} \frac{\mu \phi_{\mathrm{we}} C_{t}}{k_{\mathrm{we}}} \frac{\partial p}{\partial t},
$$

where

$$
\begin{gathered}
\zeta=d_{f}-d-\theta+2, \\
C_{t}=C_{l}+C_{f}, \\
C_{l}=\frac{1}{\rho} \frac{\partial \rho}{\partial p}, \quad C_{f}=\frac{1}{\phi_{f}} \frac{\partial \phi_{f}}{\partial p} .
\end{gathered}
$$

Initial condition is

$$
p(r, 0)=0 \text {. }
$$

Inner boundary conditions are

$$
\begin{gathered}
\left.\left(r^{2} \frac{\partial p}{\partial r}\right)\right|_{r=r_{\mathrm{we}}}=\frac{\mu}{2 \pi k_{\mathrm{we}}}\left[B q(t)+C \frac{d p_{w}}{d t}\right] \\
p_{w}(t)=p\left(r_{\mathrm{we}}, t\right) .
\end{gathered}
$$
lowing.

The three kinds of outer boundary conditions are the fol-

Case 1. Infinite pressure outer boundary condition:

$$
p(\infty, t)=p_{0}
$$

Case 2. Constant pressure outer boundary condition:

$$
p(R, t)=p_{0}
$$

Case 3. Closed outer boundary condition:

$$
\left.\frac{\partial p}{\partial r}\right|_{r=R}=0
$$

The following dimensionless parameters can be defined in order to simplify the formulations:

$$
\begin{gathered}
p_{D}=\frac{6.409 \times 10^{-12} k_{\mathrm{we}} r_{\mathrm{we}}}{B \mu q}\left(p_{0}-p\right), \\
p_{w D}=\frac{6.409 \times 10^{-12} k_{\mathrm{we}} r_{\mathrm{we}}}{B \mu q}\left(p_{0}-p_{w}\right), \\
q_{D}(t)=\frac{q(t)}{q_{e}}, \\
t_{D}=\frac{10^{-2} k_{\mathrm{we}} t}{\mu \phi_{\mathrm{we}} C_{t} r_{w}^{2}}, \quad C_{D}=\frac{C}{6.283 \phi_{\mathrm{we}} C_{t} r_{w}^{2} r_{\mathrm{we}}}, \\
r_{D}=\frac{r}{r_{\mathrm{we}}}=\frac{r}{r_{w}} e^{S}, \quad R_{D}=\frac{R}{r_{\mathrm{we}}}=\frac{R}{r_{w}} e^{S} .
\end{gathered}
$$

By substituting the above dimensionless parameters into (B.4)-(B.10), the dimensionless mathematical model for fractal reservoir with spherical flow is given by

$$
\begin{gathered}
\frac{\partial^{2} p_{D}}{\partial r_{D}^{2}}+\frac{\zeta}{r_{D}} \frac{\partial p_{D}}{\partial r_{D}}=\frac{r_{D}^{\theta}}{e^{2 S}} \frac{\partial p_{D}}{\partial t_{D}} \\
p_{D}\left(r_{D}, 0\right)=0, \\
\frac{d p_{w D}}{d r_{D}}=-q_{D}\left(t_{D}\right)+C_{D} \frac{d p_{w D}}{d t_{D}}, \\
p_{D}\left(\infty, t_{D}\right)=0 \text { or } p_{D}\left(R_{D}, t_{D}\right)=0 \\
\text { or }\left.\frac{\partial p_{D}}{\partial r_{D}}\right|_{r_{D}=R_{D}}=0 .
\end{gathered}
$$




\section{Nomenclature}

B: Formation volumefactor, $\mathrm{RB} / \mathrm{STB}$

$C$ : Wellbore storage coefficient, $\mathrm{m}^{3} / \mathrm{Pa}$

$C_{f}$ : Formation compressibility, $\mathrm{Pa}^{-1}$

$C_{l}$ : Fluid compressibility, $\mathrm{Pa}^{-1}$

$C_{t}$ : Total compressibility, $\mathrm{Pa}^{-1}$

$d$ : Euclid dimension

$d_{f}$ : Fractal dimension

$k$ : Permeability, md

p: Reservoir pressure, $\mathrm{Pa}$

$q$ : Production rate or injection rate, $\mathrm{m}^{3} / \mathrm{s}$

$R$ : Radial distance of the outer boundary, $m$

$r$ : Radial distance in spherical coordinate, $\mathrm{m}$

$S$ : Skin factor

$t$ : Time, $s$

$v_{r}:$ Spherical flow velocity, $\mathrm{m} / \mathrm{s}$

$z$ : Laplace transform variable.

\section{Greek Symbols}

$\theta$ : Fractal exponent

$\mu$ : Viscosity, cp

$\phi$ : Porosity.

\section{Subscripts}

\section{D: Dimensionless \\ $o$ : Initial \\ $w$ : Wellbore parameter \\ we: Wellbore effective.}

\section{Acknowledgments}

The authors would like to thank the anonymous referee for his/her helpful suggestions and comments. The research is supported by Sichuan Provincial Youth Science and Technology Fund (no. 2012JQ00 10), Program for New Century Excellent Talents in University (no. NCET-11-1062), and PetroChina Innovation Foundation (no. 2011D-5006-0201).

\section{References}

[1] M. H. Jia and S. C. Li, "The similar structure of solution of differential equation on boundary value problem," Journal of College Mathematics, vol. 21, no. 5, pp. 37-39, 2005.

[2] P. S. Zheng, S. C. Li, and Y. F. Zhang, "The formal similarity of solutions on the class of ordinary differential equation system," Journal of Jilin University, vol. 23, no. 8, pp. 56-60, 2005.

[3] P. S. Zheng, S. C. Li, and Y. F. Zhang, “The solution's structure of a type of ordinary differential equation system with closed right boundary conditions," Journal of Xihua University, vol. 24, no. 6, pp. 88-90, 2005.

[4] Z. C. Chen, P. H. Liu, and S. C. Li, "The similar structure of composite Bessel equation on fixed solution problem," Journal of Chongqing Techno Business University, vol. 23, no. 1, pp. 1-4, 2006.

[5] S. C. Li, L. Z. Yi, and P. S. Zheng, "The similar structure of differential equation on fixed solution problem," Journal of Sichuan
University, vol. 43, no. 4, pp. 933-934, 2006.

[6] S. C. Li, "The similar structure of solution of second-order linear homogeneous differential equation with constant coefficients on the boundary value problem," Journal of Xihua University, vol. 26, no. 1, pp. 84-85, 2007.

[7] S. C. Li, "The similar structure of solutions to the boundary value problem for second-order linear homogeneous differential equation," Journal of Xihua University, vol. 28, no. 5, pp. 4041, 2009.

[8] J. Yuan, S. C. Li, and C. L. Xing, "The structures of solutions to first class boundary value problem of second-order Euler's equation," Journal of Xihua University, vol. 28, no. 6, pp. 86-88, 2009.

[9] J. Chang and Y. C. Yortsos, "Pressure-transient analysis of fractal reservoirs," SPE Formation Evaluation, vol. 5, no. 1, pp. 31-38, 1990.

[10] R. A. Beier, "Pressure-transient model for a vertically fractured well in a fractal reservoir," SPE Formation Evaluation, vol. 9, no. 2, pp. 122-128, 1994.

[11] R. Camacho-Velázquez, G. Fuentes-Cruz, and M. VásquezCruz, "Decline-curve analysis of fractured reservoirs with fractal geometry," SPE Reservoir Evaluation and Engineering, vol. 11, no. 3, pp. 606-619, 2008.

[12] M. Cossio, "A semi-analytic solution for flow in finite-conductivity vertical fractures using fractal theory," in Proceeding of the SPE Annual Technical Conference and Exhibition, San Antonio, Tex, USA, October 2012.

[13] S. C. Li, P. S. Zheng, and Y. F. Zhang, Similar Structure of WellTest Analytical Solution in the Fractal Reservoir, China Science and Technology Press, Beijing, China, 2005.

[14] S. C. Li and J. J. Zhang, "Similar structure of pressure distribution in the fractal double porosity reservoir," Journal of Xihua University, vol. 25, no. 1, pp. 40-43, 2006.

[15] W. B. Zhu, S. C. Li, and C. X. Xu, "Similar structure of well-test analytical solution in the fractal multilayer reservoir," Drilling \& Production Technology, vol. 31, no. 3, pp. 67-69, 2007.

[16] C. X. Xu, S. C. Li, and W. B. Zhu, "Similar structure of well-test analytical solution in the fractal composite reservoir," Drilling \& Production Technology, vol. 29, no. 5, pp. 39-42, 2006.

[17] I. Ershaghi, S. W. Rhee, and H. T. Yanq, "Analysis of pressure transient data in naturally fractured reservoirs with spherical flow," in Proceeding of the 51st Annual Fall Technical Conference and Exhibition of the Society of petroleum Engineers of AIME, New Orleans, La, USA, October 1976.

[18] M. A. Proett and W. C. Chin, "New exact spherical flow solution with storage and skin for early-time interpretation with applications to wireline formation and early-evaluation drillstem testing," in Proceedings of the SPE Technical Conference and Exhibition, pp. 463-478, Louisiana, New Orleans, La, USA, September 1998.

[19] C. C. Sheng, S. C. Li, and L. J. Guo, "Similar structure of solutions for spherical flow homogeneous fractal oil reservoir," Journal of Lanzhou University of Technology, vol. 37, no. 6, pp. 136-139, 2011.

[20] C. C. Sheng, S. C. Li, and D. X. Xu, "Fractal sphere seepage flow oil-gas reservoir model and its solution," Oil and Gas Field Development, vol. 29, no. 6, pp. 55-58, 2011.

[21] C. C. Sheng, S. C. Li, and X. T. Bao, "Effective wellbore radius model of fractal sphere seepage flow for an oil-gas reservoir and its solution," Journal of Xihua University, vol. 30, no. 5, pp. 29-31, 2011. 
[22] C. C. Sheng, S. C. Li, W. Li, J. C. Wang, and Y. R. He, "Fractal sphere seepage flow model and its similar structure solution," Drilling \& Production Technology, vol. 34, no. 4, pp. 35-38, 2011.

[23] C. C. Sheng, S. C. Li, Q. Y. Li, and X. X. Xiao, "Effective wellbore radius model for fractal reservoir with spherical flow and its similar structure of solution," Journal of Harbin University of Science and Technology, vol. 17, no. 3, pp. 37-41, 2012.

[24] Z. R. Cheng and S. C. Li, "The similar structure method solving the boundary value problem of Bessel equations," Journal of Sichuan Normal University, vol. 34, no. 6, pp. 850-853, 2011.

[25] W. Li, X. P. Li, S. C. Li, and Q. Y. Li, “The similar structure of solutions in fractal multilayer reservoir including a quadratic gradient term," Journal of Hydrodynamics, vol. 24, no. 3, pp. 332338, 2012.

[26] S. C. Li and X. Q. Wu, "Several important properties for the boundary value problems of second-order linear homogeneous differential equation," Journal of Xihua University, vol. 32, no. 1, pp. 23-26, 2013.

[27] H. Stehfest, "Numerical inversion of Laplace transforms," Communications of the ACM, vol. 13, no. 1, pp. 47-49, 1970.

[28] X. Y. Kong, Higher Seepage Mechanics, China Science and Technology Press, Hefei, China, 1999.

[29] J. A. Acuña, I. Ershaghi, and Y. C. Yortsos, "Practical application of fractal pressure-transient analysis in naturally fractured reservoirs," SPE Formation Evaluation, vol. 10, no. 3, pp. 173-179, 1995. 


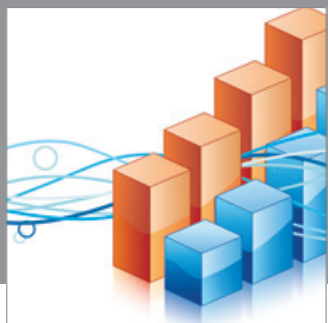

Advances in

Operations Research

mansans

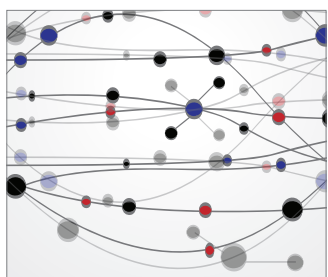

The Scientific World Journal
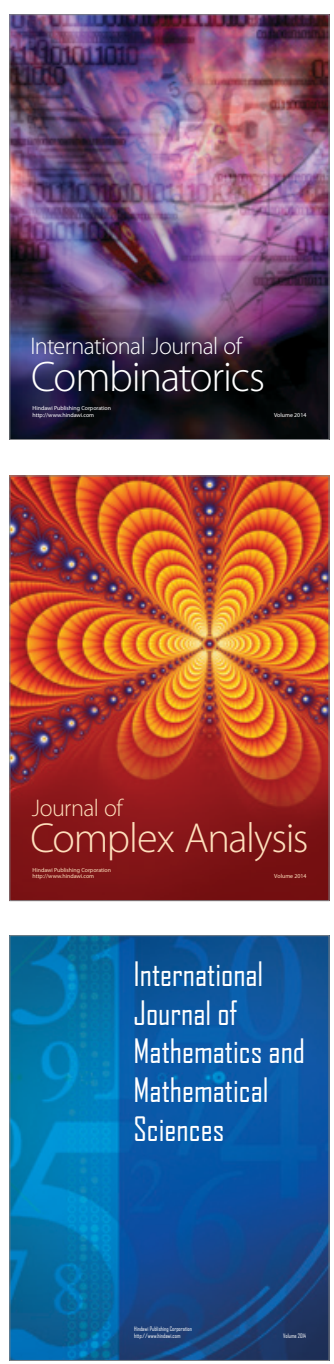
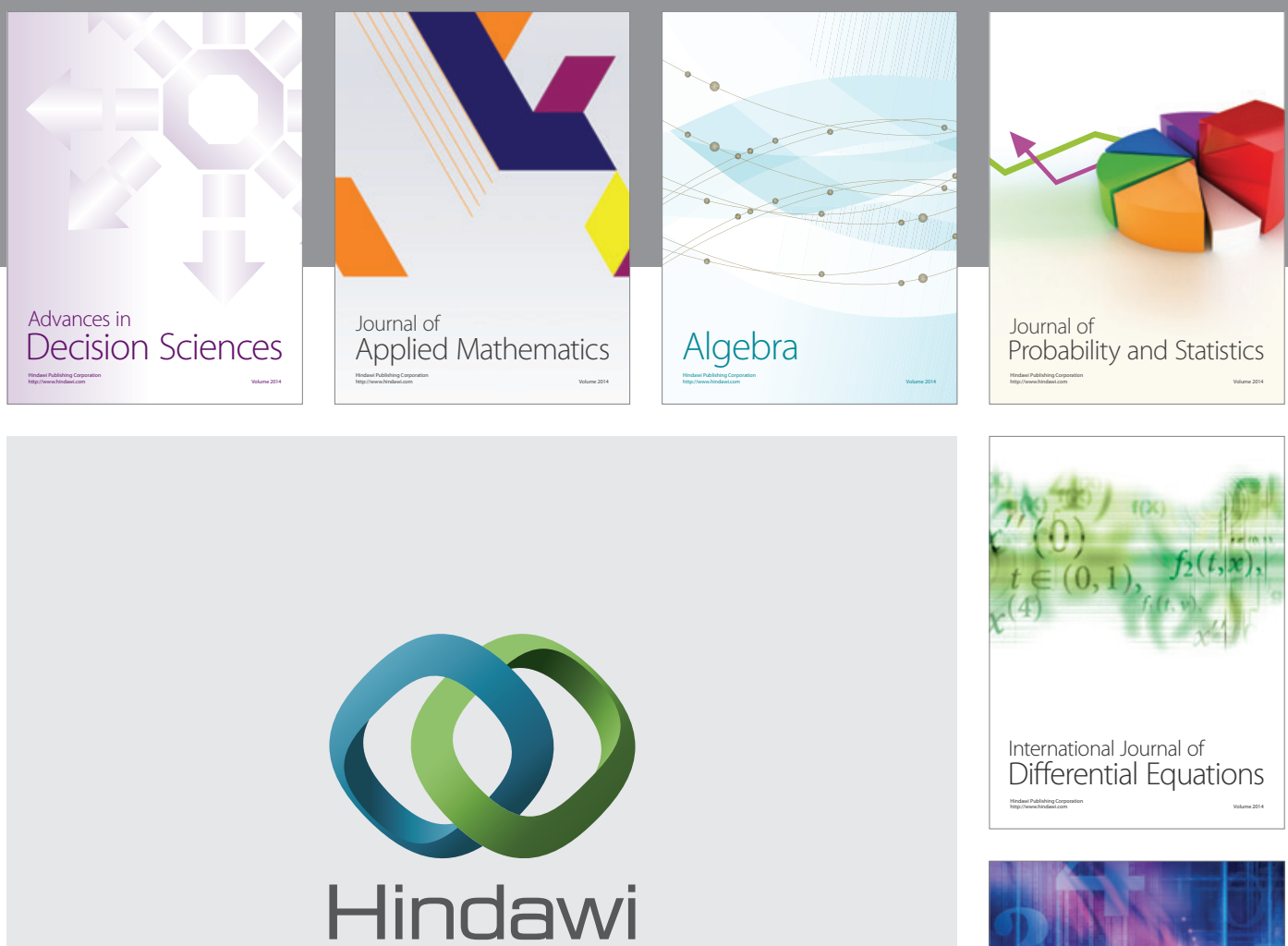

Submit your manuscripts at http://www.hindawi.com
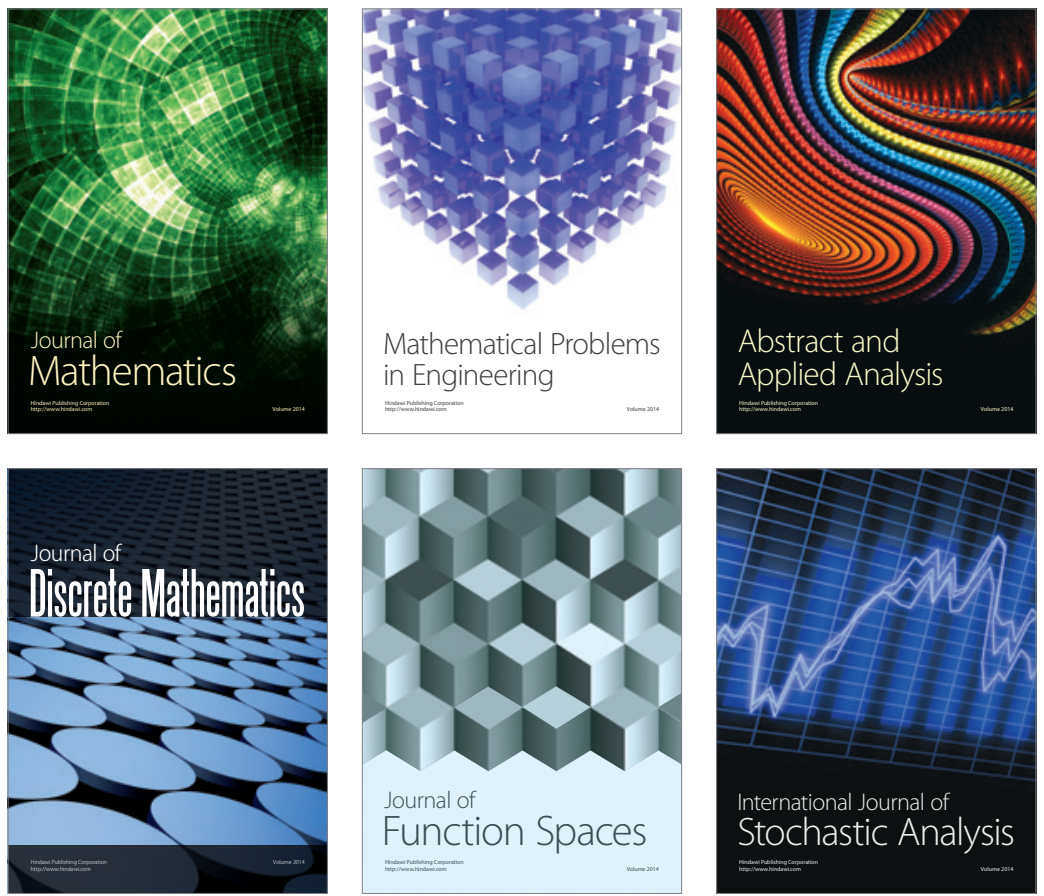

Journal of

Function Spaces

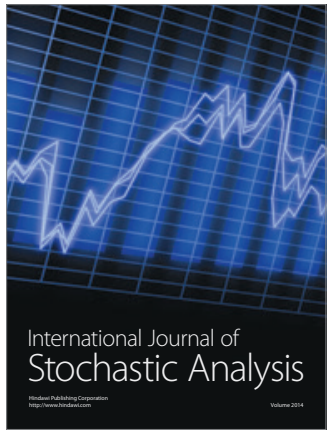

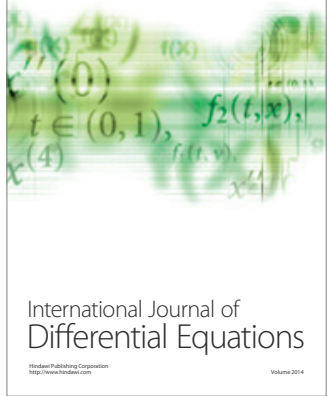
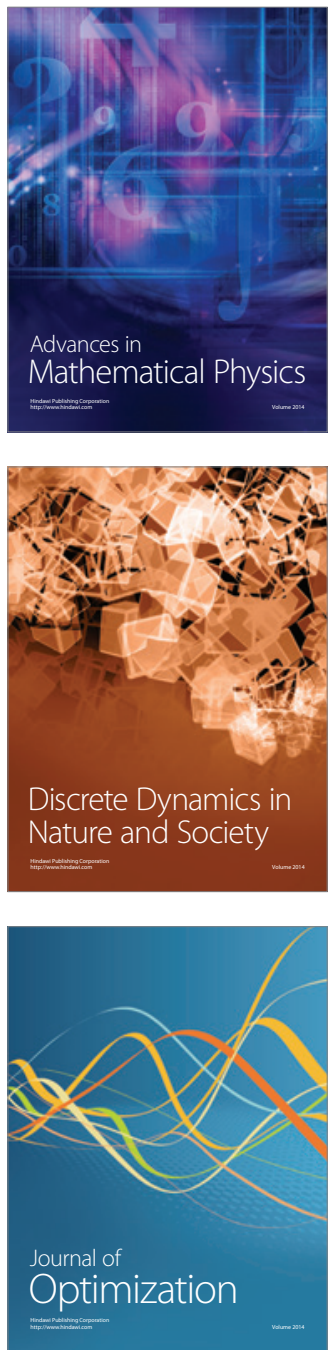